\title{
Evaluación del resultado de las acciones de detección, diagnóstico y tratamiento del Episodio Depresivo realizada en pacientes consultantes en el primer nivel de atención de la Provincia de Concepción, Chile
} Evaluation of the results of the actions of detection, diagnosis and treatment of the Depressive Episode carried out in patients attended in primary care from the Province of Concepción, Chile

Gonzalo Navarrete R. ${ }^{1,2}$, Sandra Saldivia B. ${ }^{1,2,3}$, Benjamín Vicente P. ${ }^{1,2}$ y Claudio Bustos N. ${ }^{1,3,4,5}$

\begin{abstract}
The Chilean Ministry of Health implemented since 2001 the program for treating depression in primary health care (PCH). General objective: To evaluate the result of detection, diagnostic and treatment of depressive episodes performed by consultants of the first level of care in the Province of Concepcion, Chile. Method: Follow-up of six months of a random sample of users consulting for general morbidity in PHC of the Province of Concepcion, Chile. A total of 307 users with positive diagnosis of depression (DSM-IV) using the psychiatric structured Composite International Diagnostic Interview 2.1 (CIDI) were admitted to the study. Regression analyses, binary and ordinal logistic were performed to predict the detection of the disorder, the type of treatment applied and the course of the disease, from sociodemographic, clinical and structure variables. Results: The prevalence of six months of depression reached $16.5 \%(n=431)$, prior to exclusion criteria. Doctors detected the $31.6 \%$ of the cases, with significant differences per center. Most of the interventions were of the psychosocial type (18.6\%), followed by pharmacological interventions (9.4\%) and 3.6\% of both types. After six months, the diagnosis had remitted in 60-7\% of the users. However, this evolution was not linked to the interventions received or previous diagnosis, but to personal variables of the users. Conclusions: The detection capacity of the professionals in PHC is low, interventions are scarce and probably, the evolution of the diagnosis is not affected by the intervention.
\end{abstract}

Key words: Evaluation, depression, primary care health, treatment.

Rev Chil Neuro-Psiquiat 2017; 55 (3): 160-169

Recibido: 03/05/2017

Aceptado: 25/06/2017

Los autores no presentan ningún tipo de conflicto de interés. Declaramos no haber recibido apoyo financiero de ningún tipo.

Departamento de Psiquiatría y Salud Mental. Facultad de Medicina. Univesidad de Concepción. Concepción, Chile.

PhD.

Psicólogo (a).

Mg.

$\mathrm{PhD}^{\circ}$. 


\section{Introducción}

$\mathrm{E}$ n Chile la depresión alcanza alrededor de un $10 \%$ en la población general, siendo dos veces más frecuentes en mujeres que en hombres ${ }^{1}$. Según el estudio de carga de enfermedad en Chile, del Ministerio de Salud de Chile (MINSAL), publicado el año $2007^{2}$, la depresión ocupa el segundo lugar después de las patologías cardiovasculares, entre las principales causas de discapacidad medida a través de los Años de Vida Ajustados por Discapacidad (AVISA).

Por otra parte, los países con una atención primaria en salud (APS) fuerte, exhiben una mejor situación de salud y un menor gasto, permitiendo impactar en la población de manera sistemática y universal $^{3}$, dado que es la puerta de entrada de los consultantes del sistema de salud estatal. Si consideramos el alto riesgo de recurrencia en cuadros depresivos, es imprescindible la necesidad de intervenir en el primer nivel de atención, más aun cuando existe evidencia de que la recurrencia del episodio depresivo es especialmente prevalente en aquellos pacientes tratados de modo inapropiado y/o con dosis inadecuadas y/o por un período demasiado corto ${ }^{4}$.

En el año 2000 el Ministerio de Salud de Chile puso en marcha el "Programa para la detección, diagnóstico y tratamiento integral de la depresión en atención primaria" (PDTID) ${ }^{5}$.

La primera evaluación de procesos del programa fue realizada por Alvarado et al. $(2005)^{6}$, quienes identificaron un aceptable cumplimiento de los objetivos pero, hicieron hincapié en la necesidad de desarrollar estrategias que tendieran a mejorar el diagnóstico y cumplimiento de los tratamientos ofrecidos. La segunda evaluación de procesos del programa $^{7}$ evidenció una tendencia a subcalificar la gravedad del episodio, señaló que había una aplicación "escalonada" de las intervenciones, altas tasas de abandono y falta de capacitación continua. Es importante plantear que en ambos estudios no se estableció una relación entre intervenciones y el resultado de estas, ni tampoco se hizo un análisis estructural.

En esa línea, la presente investigación buscó evaluar el resultado de las acciones de detección, diagnóstico y tratamiento del Episodio Depresivo realizadas en usuarios consultantes en 10 centros de atención primaria en salud de la Provincia de Concepción, Chile.

\section{Material y Método}

Estudio tipo descriptivo, observacional, longitudinal. Su universo lo constituyen los consultantes por morbilidad de 15 a 75 años, que acuden a los centros APS de la Provincia de Concepción. Fueron seleccionados aleatoriamente 10 del total de centros de los Servicios de Salud Concepción (SSC) y Talcahuano (SST). La muestra original fue de 3.000 usuarios. Esta se obtuvo del proyecto FONDEF (DO2I-1140) ${ }^{\text {a }}$ La muestra fue aleatoria y proporcional a la población de referencia de cada centro. Para la obtención de las modalidades de cuidados de los centros se utilizó el ICMCH (International Classification of Mental Healht Care $)^{8}$, además, de datos sociodemográficos y estructurales de ambos servicios de salud ${ }^{9}$.

Los criterios de inclusión fueron el Diagnóstico de Episodio Depresivo Mayor (DSM-IV) ${ }^{\mathrm{b}}$ y edad entre 15-75 años. Los criterios de exclusión fueron: comorbilidad con un trastorno mental grave, dependencia a sustancias (según CIDI 2.1.) $)^{c}$ y estar en tratamiento por depresión en los últimos 6 meses.

\section{Procedimiento}

Se realizó un seguimiento de 1 año, con aplicaciones a los 6 y 12 meses de CIDI 2.1 por entrevistadores capacitados. Con la submuestra, con diagnóstico de episodio depresivo mayor, se procedió a recolección de datos de la historia clínica con la ficha de recolección de información entre abril de 2004 y enero de 2005.

\footnotetext{
a "Inventario Multifactorial de Riesgo: un instrumento para una mejor predicción de futuros episodios de depresión en el nivel primario de atención".

b Diagnostic and Statitical Manual of Mental Disorders. Versión IV.

c Composite International Diagnostic Interview. Versión 2.1. (Organización Mundial de la Salud).
} 


\section{Variables}

Sociodemográficas: género, edad, estado civil, educación, migración, ocupación e ingresos; clínicas (intervenciones): farmacológicas, psicológicas, grupales y sin intervenciones, diagnóstico episodio depresivo mayor en línea base y diagnóstico episodio depresivo mayor a los 6 meses (variable dependiente). De los centros: servicio de salud, modalidades de cuidado y tasa de profesionales.

\section{Análisis de datos}

El ingreso de datos fue a través de Epiinfo (versión 5.0) y procesados en SPSS 15.0, Stata/SE 11.0 y $R$ 3.2.1. Se utilizaron frecuencias simples y porcentajes (diagnóstico y todas las variables sociodemográficas), además, de una tabla contingencia entre estas variables y la gravedad del episodio depresivo en línea de base. Para la detección: Tabla contingencia entre servicios y detección del diagnóstico; prueba exacta de Fisher diferencias significativas entre centros; y un modelo de regresión logística (episodio en línea de base, variables sociodemográficas y características de centros). Para las intervenciones: Frecuencias simples y porcentajes de tipo de intervenciones por centro; el análisis de las variables independientes que determinan el tipo de intervención ejecutada, se realizó usando tres modelos logísticos para las diferentes intervenciones; se requirió de procedimiento stepwise para obtener mínimo número de variables que resulten buenas predictoras $\left(\mathrm{AIC}^{\mathrm{d}}\right)^{10}$. Para el diagnóstico del episodio depresivo a los seis meses: Se analizaron las variables independientes usando regresión ordinal logit; se probaron tres modelos anidados, usando el diagnóstico; el diagnóstico más la intervención, y uno final con la intervención, el diagnóstico y el efecto de interacción entre ambos. También un modelo completo con todas las variables. Al no ser significativo se realizó procedimiento stepwise.

\section{Aspectos éticos}

Todos los participantes del estudio fueron sujetos voluntarios, lo cual se objetivó a través de un consentimiento informado. En la toma de datos

d Akaike Information Criterion. clínicos no hubo contacto con los usuarios. Al finalizar la toma de datos los usuarios, detectados con diagnóstico de episodio depresivo mayor, fueron informados de esta situación.

\section{Resultados}

De los 3.000 usuarios ingresados al estudio, 431 tenían diagnóstico positivo de un episodio depresivo mayor, lo que representa un 14,3\%. De ellos 307 (9,2\%) cumplían criterios de inclusión y exclusión.

En relación a los casos detectados en el SSC se observan tasas de detección entre el 21,1\% y el $50 \%$, mientras que en el SST éstas no superan el $23 \%(\mathrm{p}=0,003)$.

La muestra estaba compuesta por 46 hombres (15\%) y 261 mujeres (85\%). En las mujeres destaca el episodio depresivo severo en un $42,9 \%$ (112). La distribución de la muestra, según edad, se centró en el rango entre 25 y 64 años, con 238 usuarios (77,6\%). Del total de la muestra, 145 usuarios estaban casados $(47,2 \%)$ y 162 sin pareja $(52,8)$ entre viudos, divorciados y solteros. En relación a la variable educación, la muestra se distribuyó en la educación básica, 153 usuarios (49,8\%) y media con 128 usuarios (41,7\%). Un 73,3\% de la muestra no migró del campo a la ciudad. En cuanto a la ocupación, se centró en 172 usuarias que eran dueñas de casa (56\%). Según los ingresos, los usuarios se distribuyeron principalmente bajo el rango entre \$51-\$ 150 mil, con 210 usuarios $(68,6 \%)$.

No se identificó ningún factor del usuario que determinara la capacidad de detección. Con respecto a los factores de los centros, y al analizar las modalidades de cuidado de los dispositivos de salud $^{9}$, se observa una relación estadísticamente significativa entre la detección y el indicador intervenciones dirigidas a familia, parientes y otros $(\mathrm{p}=0,028)$. Se ajustó un modelo de regresión logístico (Tabla 1) que señala que en el SST, la probabilidad de ser detectado es menor de 0,21. La presencia de intervenciones dirigidas a familias, parientes y otros, aumenta en 2,37 los odds de ser detectados. Finalmente, un aumento de un punto 
Tabla 1. Modelo de predicción de detección del episodio depresivo

\begin{tabular}{|c|c|c|c|c|c|c|}
\hline \multirow{2}{*}{$\begin{array}{l}\text { Variable } \\
\text { Constante }\end{array}$} & \multirow{2}{*}{$\begin{array}{c}\text { Estimador } \\
-0,63\end{array}$} & \multirow{2}{*}{$\begin{array}{c}\mathbf{E E} \\
1,17\end{array}$} & \multicolumn{2}{|c|}{ OR/d (IC 95\%) } & \multirow{2}{*}{$\begin{array}{c}\mathbf{Z} \\
-0,54\end{array}$} & \multirow{2}{*}{$\begin{array}{c}\text { Valor } \mathbf{p} \\
0,59\end{array}$} \\
\hline & & & 0,53 & $(0,05-5,29)$ & & \\
\hline \multicolumn{7}{|l|}{ Factores consultorio } \\
\hline Servicio Salud $=$ Talcahuano & $-1,55$ & 0,43 & 0,21 & $(0,09-0,5)$ & $-3,59$ & $<0,01^{* *}$ \\
\hline Intervenciones dirigidas a la familia, parientes y otros & 0,86 & 0,32 & 2,37 & $(1,27-4,42)$ & 2,70 & $0,01^{* *}$ \\
\hline Tasa de médicos & $-0,58$ & 1,18 & 0,56 & $(0,06-5,69)$ & $-0,49$ & 0,63 \\
\hline Tasa de otros profesionales & $-3,17$ & 0,95 & 0,04 & $(0,01-0,27)$ & $-3,34$ & $<0,01^{* *}$ \\
\hline \multicolumn{7}{|l|}{ Factores sociodemográficos } \\
\hline Sexo $=$ Femenino & 0,32 & 0,52 & 1,37 & $(0,5-3,78)$ & 0,61 & 0,54 \\
\hline Edad & $-0,02$ & 0,01 & 0,98 & $(0,96-1,01)$ & $-1,35$ & 0,18 \\
\hline $\mathrm{EC}=$ Viudo & 0,07 & 0,43 & 1,08 & $(0,46-2,5)$ & 0,17 & 0,87 \\
\hline $\mathrm{EC}=$ Separado, Divorciado & $-0,11$ & 0,42 & 0,89 & $(0,39-2,04)$ & $-0,27$ & 0,79 \\
\hline $\mathrm{EC}=$ Soltero & $-0,63$ & 0,42 & 0,53 & $(0,23-1,21)$ & $-1,51$ & 0,13 \\
\hline Educ $=$ Ed. Básica & $-0,55$ & 0,80 & 0,58 & $(0,12-2,77)$ & $-0,69$ & 0,49 \\
\hline Educ $=$ Ed. Media & $-0,83$ & 0,84 & 0,43 & $(0,08-2,24)$ & $-1,00$ & 0,32 \\
\hline Educ $=$ Ed. técnica & $-0,07$ & 1,06 & 0,93 & $(0,12-7,43)$ & $-0,07$ & 0,94 \\
\hline Educ $=$ Ed. Superior & $-1,50$ & 1,53 & 0,22 & $(0,01-4,48)$ & $-0,98$ & 0,33 \\
\hline Migración = Sí & 0,33 & 0,34 & 1,40 & $(0,72-2,71)$ & 0,98 & 0,32 \\
\hline Ocupación $=$ Pensionado o Jubilado & $-1,12$ & 0,66 & 0,33 & $(0,09-1,2)$ & $-1,69$ & 0,09 \\
\hline Ocupación $=$ Desempleado & 0,33 & 0,60 & 1,39 & $(0,43-4,54)$ & 0,55 & 0,59 \\
\hline Ocupación $=$ Trabajador activo & $-0,07$ & 0,38 & 0,94 & $(0,44-1,97)$ & $-0,18$ & 0,86 \\
\hline Ocupación = Estudiante & $-0,35$ & 0,91 & 0,70 & $(0,12-4,18)$ & $-0,39$ & 0,70 \\
\hline Ingreso $=51-150$ & $-0,62$ & 0,39 & 0,54 & $(0,25-1,16)$ & $-1,59$ & 0,11 \\
\hline Ingreso $=151-250$ & $-0,42$ & 0,46 & 0,66 & $(0,27-1,61)$ & $-0,92$ & 0,36 \\
\hline Ingreso $=251-350$ & $-0,13$ & 0,60 & 0,88 & $(0,27-2,86)$ & $-0,21$ & 0,83 \\
\hline Ingreso $=351-450$ & 0,33 & 1,09 & 1,39 & $(0,16-11,73)$ & 0,30 & 0,76 \\
\hline Nivel depresión = Moderado & 0,41 & 0,36 & 1,51 & $(0,74-3,07)$ & 1,14 & 0,26 \\
\hline Nivel depresión = Severo & $-0,26$ & 0,34 & 0,77 & $(0,39-1,49)$ & $-0,78$ & 0,44 \\
\hline
\end{tabular}

Nota: Constante representa a Servicio Salud Concepción, con una tasa media de médicos $(\mathrm{M}=0,15)$ y otros profesionales $(M=0,25)$, en personas de sexo masculino, de edad igual a la media $(M=49,84)$, casados, sin escolaridad, que no han migrado, dueño de casa, con ingresos menores a \$50, con un nivel de depresión leve.

en la tasa de médicos sobre la media disminuye a un 0,56 los odds de ser detectados, así como el aumento en un punto en la tasa de otros profesionales sobre la media disminuye a un 0,04 los odds de ser detectados.

En los 97 pacientes diagnosticados, las intervenciones fueron simples principalmente $(73,2 \%)$, en donde un $38,1 \%$ (37) recibieron terapia grupal, a un $29,9 \%$ (29) se les indicó fármacos y un 5,2\% (5) tuvieron terapia psicológica. Los 26 restantes $(26,8 \%)$ recibieron intervenciones asociadas, en su mayoría terapia psicológica y grupal $(15,5 \%$, $\mathrm{n}=15)$.
Se analizó la posible relación entre cada uno de los tres tipos de intervención con cada una de las 4 modalidades de atención seleccionadas. Sólo la presencia de intervenciones dirigidas a la familia, parientes y otros, se relaciona de forma estadísticamente significativa con la presencia de terapias grupales $(\mathrm{p}=0,036)$.

Se ajustó un modelo de regresión logístico por tipo de tratamiento que considera las tasas de médicos, psicólogo y otros profesionales, así como los datos demográficos de las personas y la severidad del episodio depresivo.

El modelo de predicción de intervención 
farmacológica fue estadísticamente significativo $\chi^{2}(19)=38,53, \mathrm{p}=0,005$, lo que indicó que las variables predicen el hecho de ser atendido con fármacos. Al analizar los coeficientes en la Tabla 2 se pudieron ver que los solteros y viudos tenían menos probabilidad de recibir un tratamiento farmacológico que los casados. Las personas que tenían educación básica o media completa tenían menor probabilidad de ser intervenidos con fármacos que las personas sin educación formal. Finalmente, las mujeres tenían más probabilidad de ser tratadas con fármacos que los hombres.

El modelo de predicción de intervención grupal fue estadísticamente significativo $\chi^{2}(20)=43,96$, $\mathrm{p}=0,002$, lo que indica que las variables predecían el hecho de ser atendido con psicoterapia grupal.
El modelo presentó una devianza de los residuos baja, lo que indicó ajuste del modelo a los datos $\chi^{2}(285)=267.207, p=0,77$. Al analizar los coeficientes en la Tabla 3 se puede ver que existe mayor probabilidad de ser intervenido en terapia grupal en un centro que cuenta con intervenciones dirigidas a la familia, parientes y otros, y disminuye si la tasa de otros profesionales y de psicólogos es mayor. El tener un episodio depresivo severo disminuye la probabilidad de recibir terapia grupal con respecto a los que poseen depresión leve.

El modelo de predicción de intervención psicológica no fue estadísticamente significativo $\chi^{2}(19)=21,52, p=0,31$, lo que indica que el conjunto de variables de los sujetos y del centro no permitían predecir de forma adecuada la va-

Tabla 2. Modelo de predicción de la intervención farmacológica utilizando variables del centro y personales

\begin{tabular}{|c|c|c|c|c|}
\hline \multirow{2}{*}{$\begin{array}{l}\text { Variable } \\
\text { Constante }\end{array}$} & \multirow{2}{*}{$\begin{array}{c}\text { Estimador } \\
-1,27\end{array}$} & \multirow{2}{*}{$\begin{array}{c}\text { EE } \\
1,75\end{array}$} & \multicolumn{2}{|c|}{ OR } \\
\hline & & & 0,28 & $(0,01-8,66)$ \\
\hline \multicolumn{5}{|l|}{ Variables del centro } \\
\hline Intervenciones dirigidas a la familia, parientes y otros & - & - & - & -- \\
\hline Servicio Salud $=$ Talcahuano & $-0,61$ & 0,51 & 0,54 & $(0,2-1,48)$ \\
\hline Tasa de médicos & $-0,58$ & 1,68 & 0,56 & $(0,02-14,94)$ \\
\hline Tasa de otros profesionales & $-0,25$ & 1,22 & 0,78 & $(0,07-8,48)$ \\
\hline Tasa de psicólogos & - & - & - & -- \\
\hline \multicolumn{5}{|l|}{ Variables personales } \\
\hline Sexo $=$ Femenino & 1,90 & 1,08 & 6,71 & $(0,81-55,8)$ \\
\hline Edad & 0,00 & 0,02 & 1,00 & $(0,97-1,03)$ \\
\hline $\mathrm{EC}=$ Viudo & $-1,84$ & 0,78 & 0,16 & $(0,03-0,73)^{\star}$ \\
\hline $\mathrm{EC}=$ Separado, Divorciado & $-1,00$ & 0,62 & 0,37 & $(0,11-1,24)$ \\
\hline $\mathrm{EC}=$ Soltero & $-1,43$ & 0,61 & 0,24 & $(0,07-0,8)^{\star}$ \\
\hline Educ $=$ Ed. Básica & $-2,20$ & 0,95 & 0,11 & $(0,02-0,71)^{\star}$ \\
\hline Educ $=$ Ed. Media & $-2,04$ & 1,00 & 0,13 & $(0,02-0,92)^{\star}$ \\
\hline Educ $=$ Ed. Técnica / Superior & $-0,12$ & 1,16 & 0,89 & $(0,09-8,66)$ \\
\hline Migración = Sí & $-0,40$ & 0,52 & 0,67 & $(0,24-1,83)$ \\
\hline Ocupación = Inactivo & 0,84 & 1,01 & 2,31 & $(0,32-16,58)$ \\
\hline Ocupación = Activo & 0,28 & 1,03 & 1,33 & $(0,17-10,08)$ \\
\hline Ingreso $=51-150$ & $-0,97$ & 0,52 & 0,38 & $(0,14-1,06)$ \\
\hline Ingreso $=151-250$ & $-0,75$ & 0,62 & 0,47 & $(0,14-1,59)$ \\
\hline Ingreso $=251$ o más & $-0,96$ & 0,76 & 0,38 & $(0,09-1,69)$ \\
\hline Nivel depresión = Moderado & 0,43 & 0,56 & 1,54 & $(0,52-4,59)$ \\
\hline Nivel depresión $=$ Severo & 0,52 & 0,53 & 1,69 & $(0,6-4,74)$ \\
\hline
\end{tabular}

Nota: ${ }^{\star} \mathrm{p}<0,05 ;{ }^{\star \star} \mathrm{p}<0,01$. Constante representa a Servicio Salud Concepción, con una tasa media de médicos $(\mathrm{M}=0,15) \mathrm{y}$ otros profesionales $(M=0,25)$, en personas de sexo masculino, de edad igual a la media $(M=49,84)$, casados, sin escolaridad, que no han migrado, dueño de casa, con ingresos menores a \$ 50, con un nivel de depresión leve. 
Tabla 3. Modelo de predicción de la intervención grupal utilizando variables del centro y personales

\begin{tabular}{|c|c|c|c|c|}
\hline \multirow[b]{2}{*}{ Constante } & \multirow{2}{*}{$\begin{array}{c}\text { Estimador } \\
-2,86\end{array}$} & \multirow{2}{*}{$\begin{array}{c}\text { EE } \\
1,40\end{array}$} & \multicolumn{2}{|c|}{ OR } \\
\hline & & & 0,06 & $(0-0,88)$ \\
\hline \multicolumn{5}{|l|}{ Variables del centro } \\
\hline Intervenciones dirigidas a la familia, parientes y otros & 1,78 & 0,55 & 5,92 & $(2-17,53)^{\star *}$ \\
\hline Servicio Salud $=$ Talcahuano & - & - & - & - \\
\hline Tasa de Médicos & $-0,62$ & 1,37 & 0,54 & $(0,04-7,8)$ \\
\hline Tasa de otros profesionales & $-2,87$ & 1,13 & 0,06 & $(0,01-0,52)^{*}$ \\
\hline Tasa de psicólogos & $-3,23$ & 0,90 & 0,04 & $(0,01-0,23)^{\star *}$ \\
\hline \multicolumn{5}{|l|}{ Variables personales } \\
\hline Sexo $=$ Femenino & 0,00 & 0,50 & 1,00 & $(0,38-2,66)$ \\
\hline Edad & $-0,02$ & 0,01 & 0,98 & $(0,95-1)$ \\
\hline $\mathrm{EC}=$ Viudo & 1,03 & 0,49 & 2,81 & $(1,07-7,4)^{\star}$ \\
\hline $\mathrm{EC}=$ Separado, Divorciado & 0,35 & 0,49 & 1,42 & $(0,54-3,69)$ \\
\hline $\mathrm{EC}=$ Soltero & $-0,05$ & 0,45 & 0,95 & $(0,39-2,29)$ \\
\hline Educ $=$ Ed. Básica & 0,29 & 0,95 & 1,33 & $(0,2-8,64)$ \\
\hline Educ $=$ Ed. Media & $-0,47$ & 0,99 & 0,62 & $(0,09-4,38)$ \\
\hline Educ $=$ Ed. Técnica / Superior & $-0,67$ & 1,18 & 0,51 & $(0,05-5,19)$ \\
\hline Migración = Sí & 0,41 & 0,39 & 1,51 & $(0,71-3,22)$ \\
\hline Ocupación = Inactivo & $-1,20$ & 0,61 & 0,30 & $(0,09-1)$ \\
\hline Ocupación = Activo & $-0,53$ & 0,59 & 0,59 & $(0,18-1,88)$ \\
\hline Ingreso $=51-150$ & $-0,13$ & 0,48 & 0,88 & $(0,35-2,24)$ \\
\hline Ingreso $=151-250$ & 0,15 & 0,54 & 1,16 & $(0,4-3,37)$ \\
\hline Ingreso $=251$ o más & 1,15 & 0,65 & 3,15 & $(0,89-11,19)$ \\
\hline Nivel depresión $=$ Moderado & $-0,06$ & 0,41 & 0,94 & $(0,42-2,09)$ \\
\hline Nivel depresión = Severo & $-0,85$ & 0,39 & 0,43 & $(0,2-0,92)^{\star}$ \\
\hline
\end{tabular}

Nota: ${ }^{\star} \mathrm{p}<0,05 ;{ }^{\star *} \mathrm{p}<0,01$. Constante representa a servicios sin intervenciones dirigidas a familia, pacientes y otros, con una tasa media de médicos $(M=0,15)$, psicólogos $(M=0,66)$ y otros profesionales $(M=0,25)$, en personas de sexo masculino, de edad igual a la media $(\mathrm{M}=49,84)$, casados, sin escolaridad, que no han migrado, dueño de casa, con ingresos menores a $\$ 50$, con un nivel de depresión leve.

riabilidad de intervenciones psicológicas. Para obtener un modelo tentativo de predictores para la intervención psicológica, se realizó un procedimiento stepwise para obtener un conjunto mínimo de variables que optimizara el criterio AIC. El resultado final es un modelo de tres variables, que al observar los coeficientes del modelo en la Tabla 4 se puede ver que una mayor edad y una mayor tasa de psicólogos en el centro de salud disminuyen las probabilidades de ser intervenido psicológicamente, en tanto que el ser viudo, separado o divorciado aumenta la probabilidad de ser intervenido de este modo, con respecto a las personas casadas.

Luego de seis meses de seguimiento, observa-
Tabla 4. Modelo tentativo de predicción de la intervención psicológica

\begin{tabular}{|lccc|}
\hline Variable & Estimador & EE & OR/d \\
Constante & $-3,12$ & 0,41 & $0,04(0,02-0,1)^{* *}$ \\
Tasa de psicólogos & $-1,22$ & 0,80 & $0,29(0,06-1,4)$ \\
Edad & $-0,04$ & 0,02 & $0,96(0,92-1)^{*}$ \\
EC = Viudo & 1,45 & 0,69 & $4,26(1,1-16,44)^{*}$ \\
EC = Separado, & 1,33 & 0,58 & $3,76(1,21-11,66)^{\star}$ \\
$\begin{array}{l}\text { Divorciado } \\
\text { EC = Soltero }\end{array}$ & $-0,47$ & 0,73 & $0,62(0,15-2,61)$ \\
\hline
\end{tabular}

Nota: la variable Constante corresponde al efecto para un consultorio con tasa promedio de psicólogos $(M=0,66)$, en personas de edad igual a la media $(\mathrm{M}=49,84)$, casadas. 
Tabla 5. Pruebas de razón de verosimilitud para el diagnóstico a los 6 meses, basado en diagnóstico inicial e intervenciones realizadas

\begin{tabular}{|lcccccc|}
\hline & & Modelo nulo & \multicolumn{4}{c|}{ Diferencia con modelo siguiente } \\
Modelo & gl & $\chi^{2}$ & Valor $\mathbf{p}$ & gl & $\chi^{2}$ & Valor $\mathbf{~}$ \\
Diagnóstico inicial & 2 & 4,38 & 0,111 & - & - & - \\
Diagnóstico inicial + Intervención & 5 & 5,34 & 0,375 & 3 & 0,96 & 0,81 \\
Diagnóstico inicial x Intervención & 11 & 9,43 & 0,58 & 6 & 4,08 & 0,67 \\
\hline
\end{tabular}

mos como evolucionó el diagnóstico de episodio depresivo mayor en línea base. Un 51,8\% (159) no cumplió con criterios para un episodio depresivo a los 6 meses; es decir, la mayoría mostró una mejoría. Un 6,2\% (19) se ubicó en episodio leve, un $14 \%$ (43) en moderado y un $28 \%$ (86) tenía un episodio depresivo severo, la mitad de ellos $(\mathrm{n}=43)$ mantenía esta gravedad desde la línea de base.

Se analizó si la severidad del episodio depresivo a los 6 meses podía ser predicho sólo a partir del diagnóstico inicial y el tratamiento aplicado, y de la interacción entre ambos. Para ello, se probaron tres modelos anidados (Tabla 5): a) un primer modelo que incluyó sólo el diagnóstico inicial; b) un segundo modelo que incluyó el diagnóstico inicial, los tratamientos, sin interacción; y c) un modelo completo, que incluyó el diagnóstico inicial, los tratamientos y su interacción. Ninguno de los modelos por sí solo puede predecir el diagnóstico a los 6 meses, así como tampoco existen diferencias significativas entre ellos al aumentar su complejidad. Para obtener un modelo tentativo de predictores para el diagnóstico a los 6 meses, se realizó un procedimiento stepwise para obtener un conjunto mínimo de variables que optimizara el criterio AIC. Se obtuvo un modelo con tres predictores, que resultaron estadísticamente significativos, $\chi^{2}(7)=18.772, p=0,009$. Se pudo observar que el hecho de ser mujer, aumenta la probabilidad de que el diagnóstico se agrave al cabo de 6 meses. Por el contrario, los solteros tienen menos probabilidad de empeorar que los casados, así como las personas con educación básica tienen también menos probabilidad de empeorar que las personas sin educación.

\section{Discusión}

El elemento diferenciador de las evaluaciones previas del PDTID, es que estas se remitieron a procesos, y el presente estudio realizó un análisis de procesos, estructura y resultados.

Nuestra muestra coincide con la literatura en lo que se refiere al perfil sociodemográfico esperable $^{11-16}$. Identificamos una baja capacidad de detección del episodio depresivo, lo cual se acerca a los resultados del metaanálisis de Mitchell et al. $(2009)^{17}$, en donde la detección del episodio depresivo en atención primaria varía en rangos de $47,3 \%$ a $50,1 \%$. Si consideramos que la detección corresponde al médico, es importante evaluar cómo su alta rotación e incluso los bajos resultados de la capacitación ${ }^{18}$ inciden en ella. En cuanto a las características del usuario, ninguna característica de éste permite detectar el episodio depresivo. La detección mejoró si el centro presentaba intervenciones dirigidas a la familia, parientes y otros, que confirma la pertinencia del enfoque del Gobierno de Chile hacia un primer nivel de atención en salud sostenido en el Centro de Salud Familiar (CESFAM $)^{19}$. Esto también repercute cuando analizamos las intervenciones realizadas. Estas son escasas, predominando la terapia grupal que se asoció a la modalidad intervenciones dirigidas a la familia, parientes y otros. La intervención psicológica y farmacológica varía también según variables sociodemográficas y de los centros. Estos resultados contrastan en variados aspectos con los obtenidos en las evaluaciones del Programa para la Detección, Diagnóstico y Tratamiento de la depresión, en donde las intervenciones se entregan en todos los centros. Otro elemento es que no se entregan 
de manera escalonada de acuerdo a la gravedad del cuadro clínico y en base a las recomendaciones de la guía de práctica clínica ${ }^{6,7}$.

Nuestros resultados indican que las variables sociodemográficas que permiten predecir el diagnóstico a los seis meses son: el estado civil, el sexo y el nivel educacional. Con relación a los centros, ninguna de las características de estos permiten predecir el diagnóstico a los seis meses. Identificamos también, la falta de elementos estructurales mínimos (recursos humanos y características de los centros) para asegurar un adecuado funcionamiento. Las intervenciones realizadas no evidenciaron un efecto predictor en la presencia o ausencia del diagnóstico. La literatura nacional e internacional, mayoritariamente avala la eficacia de la asociación de intervenciones (psicológica y farmacológica) para el tratamiento de la depresión ${ }^{20-25}$. Es por ello que la baja implementación de la guía clínica es un elemento determinante en la efectividad de las intervenciones. Esto es algo ya identificado en la literatura internacional, siendo consistentes los estudios a nivel internacional que informan de importantes deficiencias en cuanto al cumplimiento de los tratamientos de salud mental ${ }^{26,27}$. Dado la Implementación de las Garantías Explícitas en Salud (GES) para la depresión, que aborda las inequidades y problemas de acceso a los cuidados en salud $^{28}$, y que el funcionamiento del programa no han variado sustancialmente, los avances deben ir destinados a fortalecer los aspectos estructurales y la calidad de las intervenciones.

\section{Agradecimientos}

Al equipo de profesionales y administrativos del Departamento de Psiquiatría y Salud Mental de la Facultad de Medicina de la Universidad de Concepción.

\section{Resumen}

El Ministerio de Salud de Chile implementó, a partir del año 2001, el programa de tratamiento de la depresión en la Atención Primaria en Salud (APS). Objetivo general: Evaluar el resultado de las acciones de detección, diagnóstico y tratamiento del episodio depresivo realizadas en consultantes del primer nivel de atención de la Provincia de Concepción, Chile. Método: Seguimiento de seis meses de muestra aleatoria de usuarios consultantes por morbilidad general en centros APS Provincia de Concepción, Chile. Se ingresaron al estudio 307 usuarios, con diagnóstico positivo de depresión (DSM-IV), usando la entrevista psiquiátrica estructurada Composite International Diagnostic Interview 2.1 (CIDI). Se realizaron análisis de regresión logística binaria y ordinal para predecir la detección del trastorno, el tipo de tratamiento aplicado y el curso de la enfermedad a partir de las variables sociodemográficas, clínicas y de estructura. Resultados: La prevalencia de seis meses de depresión fue de 16.7\% ( $n=431)$, previo criterios de exclusión. Los médicos detectaron el 31,6\% de los casos, existiendo marcadas diferencias por centro. En su mayoría las intervenciones fueron de tipos psicosociales (18,6\%), seguidas por las farmacológicas (9,4\%) y un 3,6\% con ambos tipos. Al cabo de seis meses, el diagnóstico había remitido en un $60.7 \%$ de los usuarios. Sin embargo, esta evolución no estaba asociada a las intervenciones recibidas, ni al diagnóstico previo, sino a variables personales de los usuarios. Conclusiones: La capacidad de detección de los profesionales en APS es baja, las intervenciones son escasas y no parecen incidir sobre la evolución del diagnóstico.

Palabras clave: Evaluación, depresión, primer nivel de atención, tratamiento. 


\section{Referencias bibliográficas}

1. Vicente B, Rioseco P, Saldivia S, Kohn P, Torres S. Estudio chileno de prevalencia psiquiátrica (DSMIII-R/CIDI)(ECCP). Rev Med Chile 2002; 130: 527-36.

2. Ministerio de Salud de Chile. Informe Final Estudio de carga de enfermedad y carga atribuible. 2007. Chile.

3. Téllez A. Atención primaria: factor clave en la reforma al sistema de salud. Dirección de asuntos públicos. Pontificia Universidad Católica de Chile. 2006. Chile.

4. Mueller TI, Leon AC, Keller MB, Solomon DA, Endicott J, Coryell W, et al. Recurrence after recovery from Major Depressive Disorder during 15 years of observational follow-up. American Journal of Psychiatry 1999; 156: 1000-6.

5. Ministerio de Salud de Chile. Programa Nacional de Detección, Diagnóstico y Tratamiento Integral de la Depresión en Atención Primaria. Chile. 2001. Unidad de Salud Mental.

6. $\quad$ Alvarado R, Vega J, Sanhueza G, Muñoz M. Evaluación del Programa para la detección, diagnóstico y tratamiento de la depresión en la atención primaria en Chile. Rev Panam Salud Pública 2005; 18 (4/5): 278-86.

7. Alvarado R, Rojas G. El programa nacional para el diagnóstico y tratamiento de depresión en atención primaria: una evaluación necesaria. Rev Med Chile 2011; 139: 592-9.

8. De Jong A. Development of the International Classification of Mental Health Care (ICMHC). Acta Psychiatr Scand 2002; 102 (405): 8-13.

9. Saldivia S, Grandon P, Melipillan R. Descripción y Evaluación de Servicios de Salud Mental en el Área Concepción-Talcahuano. Informe de proyecto. Dirección de Investigación Universidad del Desarrollo. 2005. 80.09.322.

10. Akaike H. A new look at the statistical model identification. Automatic Control, IEEE Transactions on. AC 1974; 9 (6): 716-23.

11. Meltzer H, Gill B, Petticrew M. OPCS Surveys of Psychiatric Morbidity in Great Britain. Report No 1. The prevalence of psychiatric morbidity among adults aged 16-64 living in private households in
Great Britain. HMSO: London. 1995.

12. Judd LL, Akiskal HS, Maser JD, Zeller PJ, Endicott J, Cryell W. Major depresive disorder: a prospective study of residual subthreshold depressive symtoms as predictor of rapid relapse. Journal Affective Disorders 1998; 50 (2-3): 97-108.

13. King M, Nazareth I, Levy G, Walker C, Morris R, et al. Prevalence of common mental disorders in general practice attendees across Europe. The British Journal of Psychiatry 2008; 192: 362-7.

14. National Institute of Mental Health (NIMH), https://www.nimh.nih.gov/health/statistics/prevalence/major-depression-among-adults.shtml.

15. Zavala-González MA, Domínguez Sosa G. Depresión y Percepción de la Funcionalidad familiar en adultos mayores urbanos mexicanos. Psicogeriatría 2010; 2 (1): 41-8.

16. Vicente B, Rioseco P, Saldivia S, Kohn P, Torres S. Estudio Chileno de prevalencia psiquiátrica (DSM-III-R/CIDI)(ECCP). Rev Med Chile 2002; 130: 527-6.

17. Mitchell AJ1, Vaze A, Rao S. Clinical diagnosis of depression in primary care: a meta-analysis. Lancet 2009; 374 (9690): 609-19.

18. Levav I, Kohn R, Palacio C, Rozic P, Vicente B. Training Latin American primary care physicians in the WPA module on depression: results of a multicenter trial. Psychol Med 2005; 35 (1): 35-45.

19. Ministerio de Salud. Resolución exenta $\mathrm{N}^{\circ} 37$. 2012 Manual de apoyo a la implementación del Modelo de Atención Integral de Salud familiar y comunitario en la red de salud.

20. Simin GE, Revicki D, Vonkorff M. Telephone assesmente of depression severity. J Psychiatr Res 1993; 27 (3): 247-52.

21. Geddes J, Butler R, Hatcher S. Depressive disorders. BMJ Publishing Group. Clinical Evidence Mental Health 2002; 84-106.

22. Araya R, Alvarado R, Minoletti A. Chile: an ongoing mental health revolution. Lancet 2009; 374: 507-98.

23. Gilbody S, Whitty P, Grimshaw J. Educational and organizational interventions to improve the management of depression in primary care: A systematic review. JAMA 2003; 289 (23): 3145-51.

24. Dietrich AJ, Oxman TE, Williams JW Jr, Schul- 
berg HC, Bruce ML, et al. Re-engineering systems for the treatment of depression in primary care: cluster randomised controlled trial. BMJ 2004; 329 (7466): 602.

25. Cuijpers P, Van Straten A, Warderman L, Anderson G. Psychological treatment versus combined treatment of depression: A meta-analysis. Depression \& Anxiety 2009; 26: 279-88.

26. Wang P, Demler O, Kessler R. Adequacy of treatmente for serious mental ilness in the United
States. American Journal of Public Health 2002; 92: 92-8.

27. Wang P, Lane M, Ofson M, Pincus H, Wells K, Kessler R. Twelve month use of mental health services in the U.S.: Results from the National Comorbidity Survey Replication (NCSR). Archives of General Psychiatry 2005; 62: 629-40.

28. Araya R, Alvarado R, Minoletti A. Chile: an ongoing mental health revolution. Lancet 2009; 374: 507-98.

Correspondencia:

Gonzalo Navarrete Ríos.

Avda. Juan Bosco s/n. Servicio de Psiquiatría.

Casilla 160-C. Facultad de Medicina. Universidad

de Concepción. Concepción. Chile.

Fono: 412312799 - 996619160.

E-mail: gonavarrete@udec.cl 